SHORT REPORT

\title{
Spinal dural arteriovenous fistulas: clinical features in 80 patients
}

\author{
K Jellema, L R Canta, C C Tiissen, W J van Rooij, P J Koudstaal, J van Gijn
}

J Neurol Neurosurg Psychiatry 2003;74:1438-1440

The aim of this study was to describe the clinical spectrum of spinal dural arteriovenous fistulas (SDAF) in a large group of patients. We studied the records of 80 patients who were diagnosed with an SDAF in six hospitals over a 15 year period (1985-2001). We extracted data on demographic variables, initial symptoms, symptoms at the time of diagnosis, level of SDAF, and medical history. Most patients were middle aged men, and most SDAF were located in the midthoracic region. The median time to diagnosis of 80 patients with an SDAF was 15 months (range 7 days197 months). The most common initial symptoms were gait disturbances (34\%), numbness (24\%), and paresthesias $(21 \%)$. At the time of diagnosis, most common symptoms were micturition problems (80\%), leg weakness (78\%), and numbness in the legs or buttocks (69\%). The combination of all three symptoms was present in $58 \%$ of patients. Any symptoms or signs related to sacral segments had developed in 67 patients (84\%). Fifteen patients (19\%) had become wheelchair bound. SDAF is difficult to diagnose, and the delay between first symptoms and treatment is often long. In middle aged men who present with disturbances of gait with ascending motor and sensory deficits, and who subsequently report impaired voiding or other sphincter disturbance, SDAF is one of the first diagnoses that should spring to mind.

$\mathrm{S}$ pinal dural arteriovenous fistula (SDAF) is the most common form of vascular abnormality in the spinal cord. ${ }^{1}$ The prognosis depends on the neurological deficit present at the time of diagnosis. ${ }^{2}$ Recognition at an early stage is therefore essential. Diagnosis, however, is particularly difficult because the clinical features are often nonspecific. We studied the medical history, clinical features and demographic characteristics in a large group of patients in order to define precisely the spectrum of the clinical syndrome.

\section{PATIENTS AND METHODS}

We retrieved the records of 80 patients from six Dutch referring and teaching hospitals, who were diagnosed with SDAF between 1985 and 2001. Information on history, neurological examination and investigations was extracted, and the interval between onset of the first symptoms and the diagnosis was calculated. The time of diagnosis was defined as the date at which SDAF was detected by means of imaging.

\section{Statistical analysis}

The SPSS 9.0 statistical package (SPSS Inc., Chicago, IL, USA) was used for statistical analysis. One way analysis of variance with post hoc $t$ tests and Bonferroni correction was used to compare groups.

\section{RESULTS}

Of the 80 patients, $66(83 \%)$ were men. The median age at the time of first symptoms was 57.6 years (range 21-78); only one patient was under 30 years of age at symptom onset. At the time of diagnosis the median age was 60.2 years (range 34-79). Patients were often in their sixth decade at the time of diagnosis (26 patients, $33 \%$ ).

\section{Diagnosis}

The median interval between onset of symptoms and the date of diagnosis was 15.0 months (range 7 days-197 months). The delay in diagnosis was less than 1 year in 30 patients $(38 \%)$, and more than 3 years in 15 patients $(19 \%)$.

Most fistulas were located in the midthoracic region (fig 1). Only two patients had an SDAF at the cervical level, and three patients had an SDAF at the sacral level.

Most patients had a single fistula, but three patients had two fistulas. The patients with two fistulas did not show a distinctive clinical picture compared with the other patients.

A magnetic resonance imaging (MRI) scan of the spinal cord was made in 69 of the 80 patients. A myelogram was performed in the other 11 patients. In two patients the MRI did not show myelopathy or an enlarged draining vein, either of which was present in all other patients.

\section{Clinical features}

Initial and subsequent symptoms

The most common early symptoms were gait disturbances, sensory disturbances and paresthesias (table 1). The delay between first symptoms and diagnosis did not depend on the nature of the initial symptoms. Gait disturbances were the initial symptom in 27 patients (34\%). Initially these manifested only after some distance (gait claudication), but as the disease progressed, walking became difficult immediately.

Sensory loss (numbness) was the initial symptom in 19 patients $(24 \%)$ : in one or both feet in nine patients, in the legs in six patients, in the perineal region in the patients, and in both arms in one patient.

Paraesthesias (mostly pins and needles, but sometimes a burning sensation) were reported by 17 patients. In 15 patients paraesthesias were confined to the feet, while in the other two they extended up to the knees.

At the time of diagnosis the most common symptoms were micturition problems (64 patients; $80 \%$ ), leg weakness (62 patients; $78 \%$ ), and sensory disturbances ( 55 patients; $69 \%$ ). Two patients experienced spontaneous and unwanted ejaculations on exercise. Any involvement of sacral segments (disturbance of micturition, faecal incontinence, sensory loss

Abbreviations: MRI, magnetic resonance imaging; SDAF, spinal dural arteriovenous fistulas 


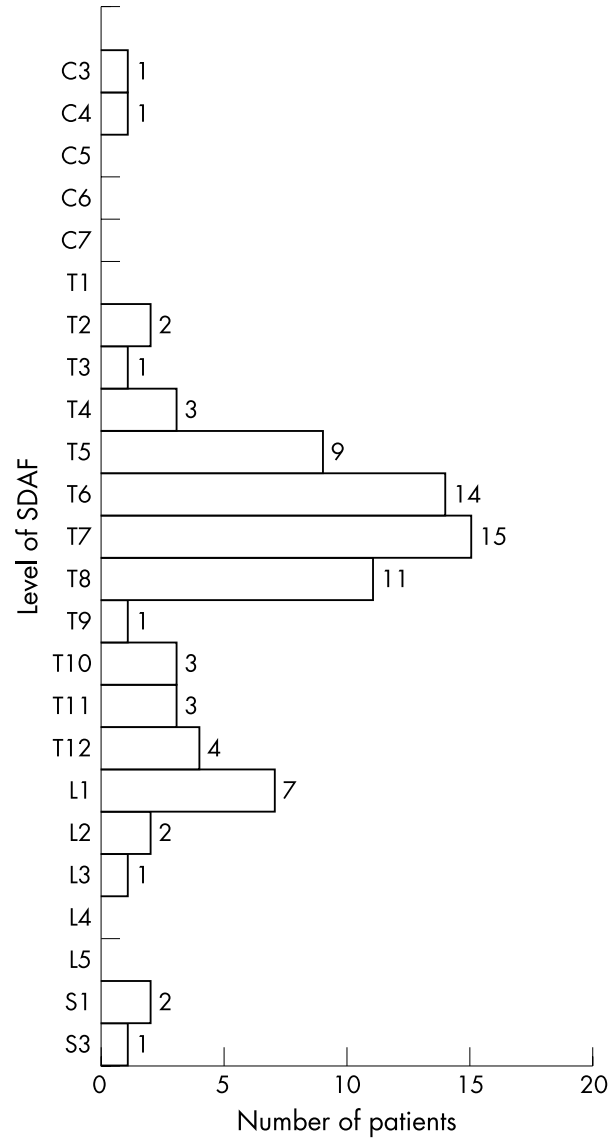

Figure 1 Most common location of 81 fistulas in 78 patients. In two patients no precise level was reported (both mid-thoracic). Three patients had two SDAFs.

in the buttocks, erectile dysfunction, or involuntary ejaculation) had occurred in 67 patients $(84 \%)$.

The most frequent combination of symptoms at the time of diagnosis (but patients could also have other symptoms) was that of micturition problems, leg weakness, and sensory disturbances of the legs (46 patients; $58 \%$ ).

\section{Motor deficits}

Altogether, 62 patients (78\%) had some degree of leg weakness at the time of diagnosis. The delay between first symptoms and diagnosis did not depend on the presence or absence of weakness. A large proportion of patients (37 patients; $47 \%$ ) suffered a mild paraparesis. At the time of diagnosis 17 patients $(21 \%)$ were wheelchair bound; in two, this resulted from previous conditions. Of the 15 patients who were wheelchair bound because of the SDAF, progression of weakness had been slow (more than 3 months) in 13, sometimes with stepwise worsening of the weakness, whereas in two patients the severe paraparesis had developed within hours or days. The interval between first symptoms and diagnosis in patients who were wheelchair bound was not significantly different from that in the others.

Upper motor neurone involvement (relatively increased tendon jerks or clonus, Babinski signs) was found in 33 patients; this was preceded by lower motor neurone involvement in 11 patients. Lower motor neurone involvement without evidence of upper motor neurone involvement was found in 29 patients.

\section{Sensory loss}

Examination at the time of diagnosis showed a sensory level to pinprick in 14 patients, and in 18 patients there was a diffuse or patchy sensory loss. Perineal sensory loss was found in 10 patients, and a stocking-like sensory loss in 13 patients. Vibration sensing in the legs was impaired in 39 patients.

\section{Rate of progression}

Overall, a gradually progressive course occurred in 50 patients $(63 \%)$. In 21 patients, episodes of acute transient deterioration were superimposed on a gradual progressive course, in five patients there was only stepwise deterioration, and four patients had an acute onset of symptoms. Acute deterioration occurred most often after exercise, but in some cases after a period of rest.

Erroneous initial diagnoses in our series of patients included, among others, sensory polyneuropathy (five patients), acute or chronic inflammatory demyelinating polyneuropathy (three patients), medullary tumour (five patients), and disc herniation (three patients).

\section{DISCUSSION}

The most striking finding in our study was that it took at least months and often years before the diagnosis of SDAF was made. The median delay was 15 months, by which time 62 of 80 patients had severe weakness of the legs and 15 had become wheelchair bound. The delay was not explained by insensitivity of MRI; in only two patients was there an absence of myelopathy or enlarged draining veins.

The difficulty in making an early diagnosis is largely explained by the rarity of the disease and the non-specific signs and symptoms (gait disturbances after exercise, or paresthesias and sensory loss in the leg), particularly in the initial phase. However, by the time the diagnosis was made, five out of six patients had one or more signs or symptoms related to the sacral segments of the spinal cord: disturbances of micturition, faecal incontinence, involuntary ejaculation, or sensory loss involving the buttocks. Involvement of sacral segments should therefore be regarded as a cardinal feature, but there are other clues as well.

\begin{tabular}{|c|c|c|c|}
\hline Initial signs and symptoms & n (\%) & Signs and symptoms at time of diagnosis & n (\%) \\
\hline Walking disturbances & $27(34)$ & Micturition problems & $64(80)$ \\
\hline Sensory disturbances & $19(24)$ & Paraparesis & $62(78)$ \\
\hline Paresthesias & $17(21)$ & Sensory disturbances & 55 (69) \\
\hline Paraparesis & $13(16)$ & Walking disturbances & $50(63)$ \\
\hline Low back pain & 11 (14) & Anal sphincter disturbances & 31 (39) \\
\hline Pain in legs & $10(13)$ & Paresthesias & 27 (34) \\
\hline Weakness of one leg & 7 (9) & Low back pain & $21(26)$ \\
\hline Cramps & $4(5)$ & Pain in legs & $20(25)$ \\
\hline Micturition problems & $3(4)$ & Erectile dysfunction & $20(25)$ \\
\hline
\end{tabular}




\section{Distinctive clinical features}

Although the clinical picture of a poly(radiculo)neuropathy may resemble that of SDAF, especially at the start of the disease, there are some important differences. First, involvement of the arms is rare in SDAF, and occurs only in cervical SDAFs, whereas most polyneuropathies are eventually associated with a stocking- and glove-like sensory loss. Secondly, the sensory loss in SDAF begins mostly distally, but over time it extends more and more proximally, ultimately to the buttocks and the perineal region. Sensory involvement of the entire leg is rare in polyneuropathy. Thirdly, micturition problems are uncommon in polyneuropathies but occur in almost $80 \%$ of patients with SDAF. Mostly the disturbance consists of urinary retention, resulting from involvement of motor, sensory and autonomic neurones in the conus medullaris. Fourthly, asymmetrical sensory or motor deficits in the legs are a frequent feature in patients with SDAF at the start of the disease, whereas in polyneuropathy symptoms are usually symmetrical. Fifthly, when upper motor neurone signs supervene (because the venous congestion in the spinal cord extends above the level of the conus medullaris) there is definitive proof that the lesion cannot be localised in roots or nerves.

\section{Rate of progression}

In most of our patients, the symptoms gradually progressed in the course of months to years, but some patients showed stepwise deterioration or an acute onset of symptoms. The sudden episodes mostly occurred after exercise or prolonged standing, and may be caused by a further increase in venous hypertension, a notion that is supported by the finding that such deteriorations may also appear during singing ${ }^{3}$ and may disappear after rest. ${ }^{4}$ An increase in venous congestion may also explain gait claudication, which is a common finding in patients with SDAF.

In conclusion, SDAF is difficult to diagnose, and the delay between first symptoms and treatment is often long. As the outcome is worse with more severe neurological deficits, attempts should be made to shorten this delay. In middle aged men who present with disturbances of gait because of ascending motor and sensory deficits, and who also report impaired voiding or any other symptom indicating dysfunction of sacral segments, SDAF is one of the first diagnoses that should spring to mind.

\section{ACKNOWLEDGEMENTS}

We would like to acknowledge the Janivo Foundation, Zeist, The Netherlands, for the funding of the current study.

\section{Authors' affiliations}

K Jellema, C C Tijssen, Department of Neurology, St. Elisabeth Hospital, Tilburg, The Netherlands

L R Canta, Department of Neurology, Máxima Medical Centre, Eindhoven, The Netherlands

W J van Rooij, Department of Neuroradiology, St. Elisabeth Hospital, Tilburg, The Netherlands

P J Koudstaal, Department of Neurology, Erasmus Medical Centre, Rotterdam, The Netherlands

J van Gijn, Department of Neurology, University Medical Centre, Utrecht, The Netherlands

Competing interest: None declared

Correspondence to: $\mathrm{K}$ Jellema, Department of Neurology, St. Elisabeth Hospital Tilburg, Hilvarenbeekseweg 60, Tilburg 5022 GC, The Netherlands; k.Jellema@elisabeth.nl

\section{REFERENCES}

1 Hurst RW, Kenyon LC, Lavi E, et al. Spinal dural arteriovenous fistula: the pathology of venous hypertensive myelopathy. Neurology 1995;45:1309-13.

2 Symon L, Kuyama H, Kendall B. Dural arteriovenous malformations of the spine. Clinical features and surgical results in 55 cases. J Neurosurg 1984;60:238-47.

3 Khurana VG, Perez-Terzic CM, Petersen RC, Krauss WE, et al. Singing paraplegia: a distinctive manifestation of a spinal dural arteriovenous fistula. Neurology 2002;58:1279-81.

4 Atkinson JLD, Miller GM, Krauss WE, et al. Clinical and radiographic features of dural arteriovenous fistula, a treatable cause of myelopathy. Mayo Clin Proc 2001;76:1120-30.

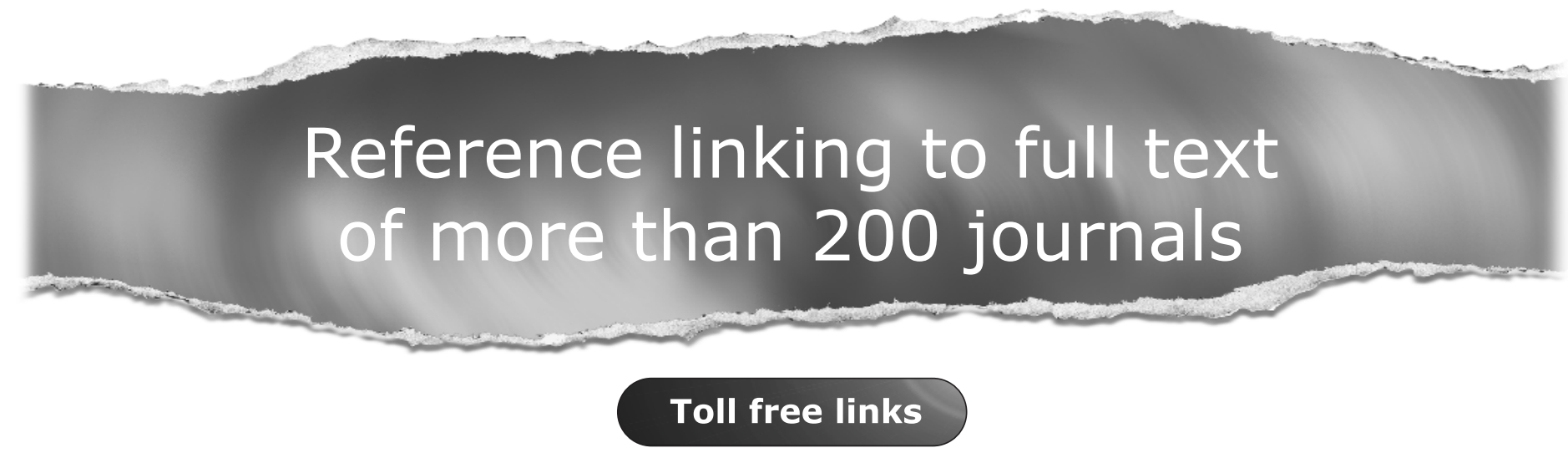

You can access the FULL TEXT of articles cited in the Journal of Neurology, Neurosurgery, and Psychiatry online if the citation is to one of the more than 200 journals hosted by HighWire (http://highwire.stanford.edu) without a subscription to that journal. There are also direct links from references to the Medline abstract for other titles.

\section{www.jnnp.com}

